\title{
Biological activities of green macroalgae enteromorpha prolifera for potential applications
}

\begin{abstract}
Enteromorpha (Ulva) prolifera, an important member of marine green algae, contains many bioactive compounds among which polysaccharides are the major components. This seaweed attracted extensive interest due to its multiple biological activities. E. prolifera has been identified as a potential producer of a wide spectrum of natural substances such as carotenoids, fucoidans and phlorotannins. These compounds show different biological activities in some vital industrial applications like pharmaceutical, nutraceutical, cosmeceutical and functional foods. This review focuses on biological activities of the green macroalgae E. prolifera based on latest research results, including antioxidant, antibacterial, immunomodulatory and hypolipidemic effects. The facts summarized here may provide novel insights into the functions of $E$. prolifera and its derivatives and potentially enable their use as functional ingredients.
\end{abstract}

Keywords: Green macroalgae; Enteromorpha prolifera; Bioactivity; Application
Volume 2 Issue 5 - 2016

\author{
Chao Zhao, ${ }^{1,2}$ Chengfeng Yang,' Bin Liu ${ }^{1,3}$ \\ 'College of Food Science, Fujian Agriculture and Forestry \\ University, China \\ 2Department of Chemistry, University of California, USA \\ ${ }^{3}$ National Engineering Research Center of JUNCAO, China
}

\begin{abstract}
Correspondence: Chao Zhao, College of Food Science, Fujian Agriculture and Forestry University, Fuzhou 350002, China, Tel 86-591-83530197, Fax 86-59I-83530197, Email zhchao@live.cn
\end{abstract}

Received: June 02, 2016 | Published: July 07, 2016

\section{Introduction}

Algae are classified as unicellular microalgae and macroalgae, which are macroscopic plants of marine benthoses. Macroalgae, also known as seaweed, are distinguished according to the nature of their pigments: brown seaweed (phaeophyta), red seaweed (rhodophyta) and green seaweed (chlorophyta). ${ }^{1}$ Seaweeds are considered as a source of bioactive compounds as they are able to produce a great variety of secondary metabolites characterized by a broad spectrum of biological activities. In the summer of 2008, the large accumulations of green macroalgal biomass occurred in the Yellow Sea brought serious ecological damage and economic losses. ${ }^{2}$ Morphological and molecular identification showed that the main species of the green tide is Enteromorpha (Ulva) prolifera, which is a macroalgae belongs to the order of Ulvales and the family of Ulvaceae (Figure 1). E. prolifera is one of the most popular green seaweeds in Asian countries. It is better known as Enteromorpha prolifera.

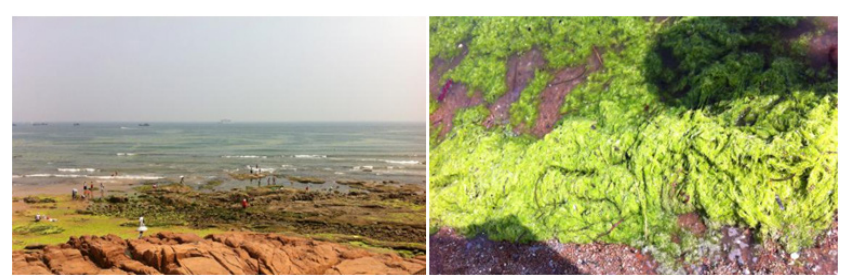

Figure I Green tide of Enteromorpha prolifera in coastal waters of the Yellow Sea near Qingdao, China, in August 2014.

\section{History, distribution and classification of enteromorpha prolifera}

There still have some controversies about division for Enteromorpha and Ulva genera. They are widely regarded as easily recognizable sea-weed genera. Despite their obvious differences in habit, they share many cellular, ultra structural, physiological, and developmental characters, including having the same type of highly tolerant and fast-adhering spores. Woolcott et al. ${ }^{3}$ were of the opinion that Enteromorpha and Ulva should be divided into two genera after studied on the rbcL gene of the East Australian Enteromorpha and
Ulva prolifera. ${ }^{3}$ However, Hayden et al. ${ }^{4}$ have conducted a systematic analysis to nearly 30 kinds of Enteromorpha and Ulva ITS nrDNA and $\mathrm{rbcL}$ gene. ${ }^{4}$ Combined with earlier molecular and culture data, they believed that there were no significant differences between these species and the two algaes should not be recognized as separate genera. All this shows that until now there has not a clear and effective standard can get Enteromorpha and Ulva completely separate.

The life history of E. prolifera is typically an alternation of isomorphic, unisexual haploid gametophytesand diploid sporophytes. This seaweed is distributed widely in the intertidal zones of shores and estuaries around the world by the virtue of its tolerance of a wide range of salinity and water temperature. It has been reported that the early germination of spores of Enteromorpha sp. required attachment to a solid substratum, such as small sand particles and the thalli, and could then grow without the need for attachment to the substratum. Santeliees et al. ${ }^{5}$ reported that Chlorophyta fragments caused by grazers or in their excreta could reproduce new individuals. ${ }^{5}$ However, little information is available about the process by which the fragments give rise to new individuals. Fragments of the appropriate from the $E$. prolifera thalli broken by a variety of factors via producing spores to give rise to the rapid proliferation of the seaweed under field conditions. $^{6}$

\section{Nutrition and medicinal value of enteromorpha in food industry}

Over the last decades, marine algae have received a lot of attention as functional food ingredients. Seaweeds have been used widely for centuries traditionally in Asia, mostly as food to provide nutrition and a characteristic taste. Fresh dried seaweed is extensively consumed, especially by people living in coastal areas. In western countries, these are used for the production of valuable chemicals and polysaccharides are the major chemical compounds. Edible chlorophyta species have $16-22.1 \%$ of protein, $12.4-18.7 \%$ of ash and $43.4-60.2 \%$ of carbohydrate as percentage of dry matter. Many studies have documented that Enteromorpha alga is famous as a nutritious and low-calorie food that is rich in essential amino acids of human, fatty acids, vitamins, dietary fibre and resistant protein, which may 
pass through the intestine without being absorbed and can retain dietary mineral components. ${ }^{7}$ Enteromorpha also contains chlorophyll $\mathrm{b}$ and many kinds of minerals, such as calcium, magnesium and iron. Chemical analysis indicated that Enteromorpha spp. has 9-14\% protein; $2-3.6 \%$ ether extract; $32-36 \%$ ash, and $n-3$ and $n-6$ fatty acids 10.4 and $10.9 \mathrm{~g} / 100 \mathrm{~g}$ of total fatty acid, respectively. ${ }^{8}$ Polysaccharide is the major component of E. prolifera. The uses of these carbohydrate polymers span from food, cosmetic and pharmaceutical industries to microbiology and biotechnology.

\section{Biological properties of enteromorpha prolifera polysaccharides}

The physical properties of polysaccharides in green seaweed are closer to those of land plant leaves than to other classes of seaweeds. Algae are a diverse group of photosynthetic organisms containing polysaccharides as the main components of biomass. In green algae, the major polysaccharides are polydisperse heteropolysaccharides where glucuronoxylorhamnans, glucuronoxylorhamnogalactans or xyloarabinogalactans are the three main groups. Many papers have been published on the chemical characterization of polysaccharides present in Ulva. However, the structures of E. prolifera polysaccharides (EPPs) present in Enteromorpha have not yet been fully characterized.

Tang et al. ${ }^{10}$ illustrated that EPPs had a high hypolipidemic activity and this activity might be due to its antioxidant potential..$^{10}$ EPPs showed decreased body weight gain, plasma triacylglycerol (TG), total cholesterol (TC), plasma low density lipoprotein cholesterol level, liver TG, liver TC and liver weight in rats. ${ }^{11}$ Cho et al. ${ }^{12}$ attributed the strong antioxidant activity of the extracts from E. prolifera to a chlorophyll compound, pheophorbide a, rather than phenolic compounds. ${ }^{12}$ EPPs exhibited potent immunomodulatory properties and could be used as a novel potential immunostimulant in food and pharmaceutical industries. ${ }^{13}$ Some research have reported that EPPs could enhance the activities of alkaline phosphatase, superoxide dismutase and lactate dehydrogenase and increase the level of NF$\square$ B. Lü et al. ${ }^{14}$ have studied antibacterial activities of EPPs and they found the stronger inhibitory of EEPs effect on Escherichia coli and better inhibitory effects on plant pathogenic fungi. ${ }^{14}$ Marine algal sulfated polysaccharides also exhibit various biological activities, including anticoagulant, antiviral, antioxidative, anticancer and antiinflammation. It was reported that sulfated polysaccharides from $E$. prolifera showed it could stimulate macrophage cells and induce considerable $\mathrm{NO}$ and various cytokine production via enhanced mRNA expression. ${ }^{15}$

\section{Conclusion}

E. prolifera contains many nutritional compounds and polysaccharides have been attracted extensive interest due to its multiple biological activities. The uses of these compounds span from food, cosmetic and pharmaceutical industries to microbiology and biotechnology. E. prolifera polysaccharides play a nutritional role as dietary fibre and demonstrate biological activities or gelling abilities. The facts summarized here may provide novel insights into the functions of E. prolifera and its derivatives and potentially enable their use as functional ingredients.

\section{Acknowledgements}

This work was financially supported by the National Natural Science Foundation of China (41306181), China Postdoctoral Science
Foundation (2014T70602), Science Foundation of the Fujian Province, China (2016J06009 \& JK2014014) and FAFU grant XJQ201417.

\section{Conflict of interest}

The author declares no conflict of interest.

\section{References}

1. Usov A, Zelinsky ND. Chemical structures of algal polysaccharides. In: H Dominguez editor. Functional Ingredients from Algae for Foods and Nutraceuticals. Chapter 2, India: Woodhead Publishing; 2013.

2. Zhao C, Ruan LW. Biodegradation of Enteromorpha prolifera by mangrove degrading micro-community with physical-chemical pretreatment. Appl Microbiol Biotechnol. 2011;92(4):709-716.

3. Woolcott GW, King RJ. Ulva and enteromorpha (ulvales, ulvophyceae, chlorophyta) in eastern australia: comparison of morphological features and analyses of nuclear rdna sequence data. Aust Syst Bot. 1999;12(5):709-725.

4. Hayden HS, Blomster J, Maggs CA, et al. Linnaeus was right all along: Ulva and Enteromorpha are not distinct genera. Eur $J$ Phycol. 2003;38(3):277-294.

5. Santeliees B, Paya I. Digestion survival of algae: some ecological comparisons between free spores and propagules in fecal pellets. $J$ Phycol. 1989;25(4):693-699.

6. Gao S, Chen X, Yi Q, et al. A strategy for the proliferation of Ulva prolifera, main causative species of green tides, with formation of sporangia by fragmentation. PLoS One. 2010;5(1):e8571.

7. Wang X, Chen Y, Wang J, et al. Antitumor activity of a sulfated polysaccharide from Enteromorpha intestinalis targeted against hepatoma through mitochondrial pathway. Tumour Biology the Journal of the International Society for Oncodevelopmental Biology and Medicine. 2013;35(2):1641-1647.

8. Aguilera-Morales M, Casas-Valdez M, Carrillo-Dominguez S, et al. Chemical composition and microbiological assays of marine algae Enteromorpha, spp. as a potential food source. J Food Compos Anal. 2005;8(1):79-88.

9. Zhao C. Purification and composition analysis of polysaccharide from edible seaweed Enteromorpha prolifera and polysaccharides depolymerized enzymes from microorganisms. Res J Biotechnol. 2014;9(1):30-36.

10. Tang Z, Gao H, Wang S, et al. Hypolipidemic and antioxidant properties of a polysaccharide fraction from Enteromorpha prolifera. Int J Biol Macromol. 2013;58:186-189.

11. Teng Z, Qian Li, Zhou Y. Hypolipidemic activity of the polysaccharides from Enteromorpha prolifera. Int $J$ Biol Macromol. 2013;62:254-256.

12. Cho ML, Lee HS, Kang IJ, et al. Antioxidant properties of extract and fractions from Enteromorpha prolifera, a type of green seaweed. Food Chem. 2011;127(3):999-1006.

13. Wei J, Wang S, Liu G, et al. Polysaccharides from Enteromorpha prolifera enhance the immunity of normal mice. Int $J$ Biol Macromol. 2014;64:1-5. 
14. Lü H, Gao Y, Shan H, et al. Preparation and antibacterial activity studies of degraded polysaccharide selenide from Enteromorpha prolifera. Carbohyd Polym. 2014;107:98-102.
15. Kim JK, Cho ML, Karnjanapratum S, et al. In vitro and in vivo immunomodulatory activity of sulfated polysaccharides from Enteromorpha prolifera. Int J Biol Macromol. 2011;49(5):10511058. 